\title{
Dielectric Properties of Multi-Layers Hexagonal Boron Nitride
}

\section{Fazail Khalid1' Ibtisam Riaz', Rashid Jalil1', Usamah Mahmood', Rehan Rahat Mir², Hafiz Amir Sohail ${ }^{1}$}

${ }^{1}$ Department of Physics, University of Engineering and Technology, Lahore, Pakistan

${ }^{2}$ Centre of Excellence in Solid State Physics, University of the Punjab, Lahore, Pakistan

Email: Fazailkhalid14@gmail.com, ibtsamriaz@gmail.com, Rashidjalil@uet.edu.pk, Ubtqazi4931992@gmail.com,

rehan.rahat.mir@gmail.com,amirsohail955@gmail.com

How to cite this paper: Khalid, M.F., Riaz, I., Jalil, R., Mahmood, U., Mir, R.R. and Sohail, H.A. (2020) Dielectric Properties of Multi-Layers Hexagonal Boron Nitride. Materials Sciences and Applications, 11, 339-346. https://doi.org/10.4236/msa.2020.116023

Received: April 21, 2020

Accepted: May 31, 2020

Published: June 3, 2020

Copyright $\odot 2020$ by author(s) and Scientific Research Publishing Inc. This work is licensed under the Creative Commons Attribution International License (CC BY 4.0).

http://creativecommons.org/licenses/by/4.0/

\begin{abstract}
A higher value of the dielectric constant of h-BN makes it quite favourable material in energy storing device. The variation in dielectric constant was observed as a function of thickness. In this research work multilayers of Hexagonal Boron Nitride (h-BN) was fabricated by using the Chemical exfoliation method. Two solvents Dimethylformamide (DMF) and Isopropyl Alcohol (IPA) were used for the exfoliation of h-BN. Successful sonication of hexagonal boron nitride led to the formation of Boron Nitride nanosheets (BNNs). The stable dispersibility of h-BN in Dimethylformamide and Isopropyl Alcohol was confirmed by UV Visible Spectroscopy, X-ray diffraction (XRD) and Scanning electron microscopy (SEM) confirm the mono crystallite structure (002) and nanoflakes like morphology of h-BN respectively. This appropriate strategy offered a feasible route to produce multilayer of hexagonal boron nitride. After the successful fabrication of h-BN multilayers its dielectric properties were calculated by using LCR meter. Profilometer revealed the variation in thickness and value of Dielectric constant was calculated by using its formula.
\end{abstract}

\section{Keywords}

Hexagonal Boron Nitride (h-BN), Boron Nitride Nanosheets (BNNs), Dimethylformamide (DMF), Isopropyl Alcohol (IPA)

\section{Introduction}

Boron Nitride is isoelectronic to carbon and it has two major allotropes: hexagonal boron nitride (h-BN) that is similar to graphite while cubic boron nitride 
(c-BN) which resembles to diamond [1]. BN is produced unnaturally by boric acid or boron trioxide. Amorphous BN powder was initially produced, that is converted to crystalline like h-BN for making it the heating process is used in the presence of nitrogen flow at above $1500^{\circ} \mathrm{C}$ temperature [2]. The h-BN is occasionally called white graphene because of its likeness and duality. It has got too much interest because of its infrequent electronic band arrangement and the nature of its charge carriers that results in high mobility and other incomparable quantum phenomena at room temperature. h-BN is a smart material because its structural properties were quite similar to graphite [3].

$\mathrm{h}-\mathrm{BN}$ has an arrangement like honeycomb lattice. It consists of $\mathrm{B}-\mathrm{N}$ rings that are covalently bonded while the hexagonal layers are bonded together by weak Vander Waals forces [4]. The interlayer space between the layers is $\sim 0.33 \mathrm{~nm}$ [5]. Two dimensional h-BN nanosheets have been intensively investigated as a class of different and unique materials, due to their fascinating and advantageous properties. Boron Nitride in its Hexagonal form, is very useful for applications as lubrication, resistant coatings, high temperature and energy storing devices. It is quite challenging to obtain controlled properties and specific structures during synthesis of h-BN.

There are some methods that were used for the fabrication of hBNNs like Mechanical Exfoliation [6], Chemical Vapour Deposition [7], and Liquid Exfoliation [8]. Liquid exfoliation is the proposed method in this paper because of its feasibility and cost effectiveness [9]. While in CVD method we need a vacuum and high temperature for the yielding of hBNNs which makes it a costly method. A lot of solvents were used for the exfoliation of h-BN as like DMF, IPA, Methanesulfonic Acid [9], Ethanol and Deionized water. The mixed solvent technique was also used for the dispersion of h-BN in which water and ethanol were mixed and used as a solvent [10]. This method has many apparent advantages, such as low cost and scalability.

\section{Materials and Experiment}

\subsection{Material}

h-BN powder (purity 97\%) was purchased by SANNO Tools. DMF and IPA were used as solvents for the exfoliation of h-BN. All chemicals were used as received. $\mathrm{Si} / \mathrm{SiO}_{2}$ was used as a substrate.

\subsection{Apparatus and Method}

A sonicator (Great sonic, power of bath $240 \mathrm{~W}$, having $50 \mathrm{~Hz}$ frequency) was used. First of all a beaker is taken which was cleaned by using acetone. After that by using digital mass balance $2 \mathrm{mg}$ of h-BN powder was measured and mixed with $50 \mathrm{~mL}$ IPA in a beaker. This solution sonicated in a sonicator for 10 Hours. Similarly, another sample was also made in which $2 \mathrm{mg}$ of h-BN powder is mixed with $50 \mathrm{~mL}$ DMF in a beaker. The solution then sonicated for the 10 hours (Figure 1). 


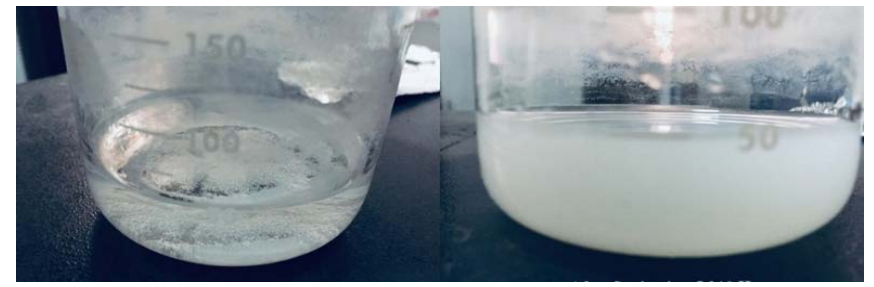

(a)

(b)

Figure 1. (a) Shows h-BN powder before Sonication while (b) shows h-BN powder after sonication.

The obtained solution was centrifuged at $3000 \mathrm{rpm}$ for $10 \mathrm{mins}$. The supernatant was collected and drop cast on silicon substrate for microscopic and spectroscopic analysis (Figure 2).

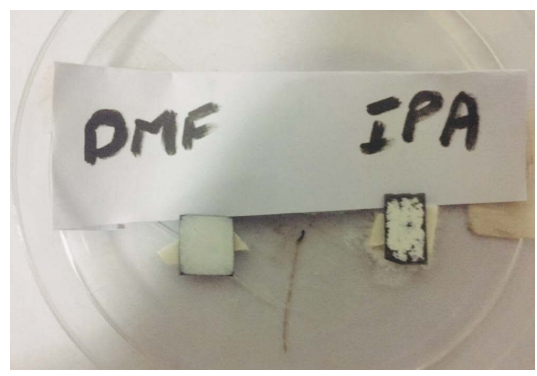

Figure 2. Shows Exfoliated h-BN in DMF and IPA drop-casted on Silicon Substrate.

\section{Results and Discussion}

\subsection{UV-Visible Spectroscopy}

One can confirm the presence of h-BN with an absorption peak in the range of 210 to $280 \mathrm{~nm}$ [11]. As the sample exfoliated in DMF having an absorption peak at $240 \mathrm{~nm}$ as shown in Figure 3(a). Similarly, another sample exfoliated in IPA which shows an absorption peak that was observed at $265 \mathrm{~nm}$ in Figure 3(b).

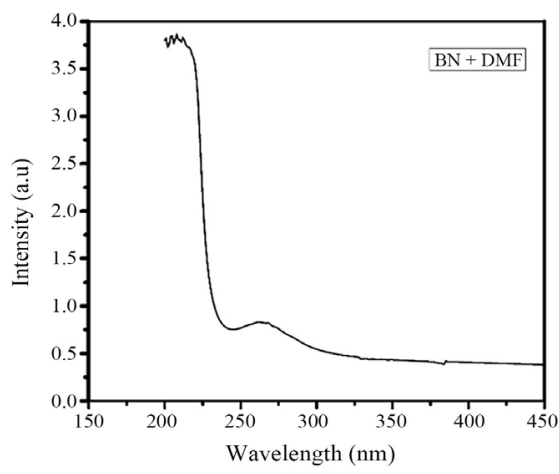

(a)

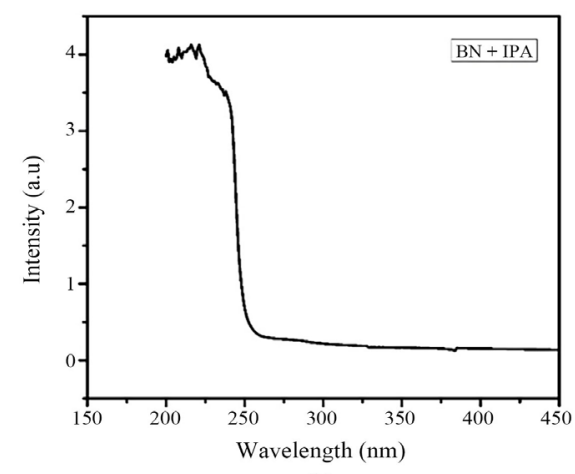

(b)

Figure 3. (a) UV-Visible spectrum of multi-layers of h-BN exfoliated in DMF; (b) UV-Visible Spectrum of h-BN exfoliated in IPA.

The multilayers of h-BN exfoliated in DMF has a bandgap of $5.39 \mathrm{eV}$ [4] while the multilayers of h-BN exfoliated using IPA as solvent has $4.96 \mathrm{eV}$ value (Figure 4). 


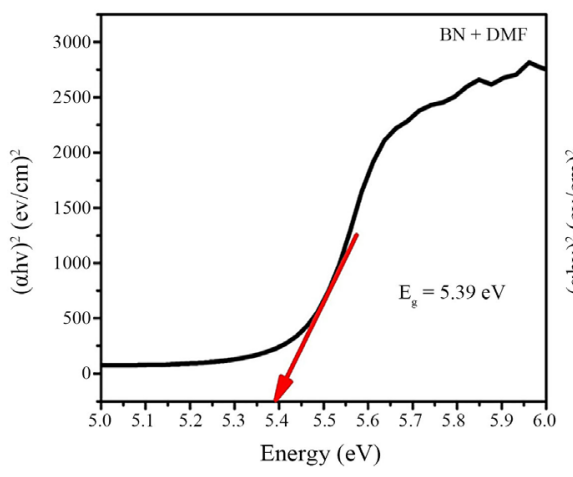

(a)

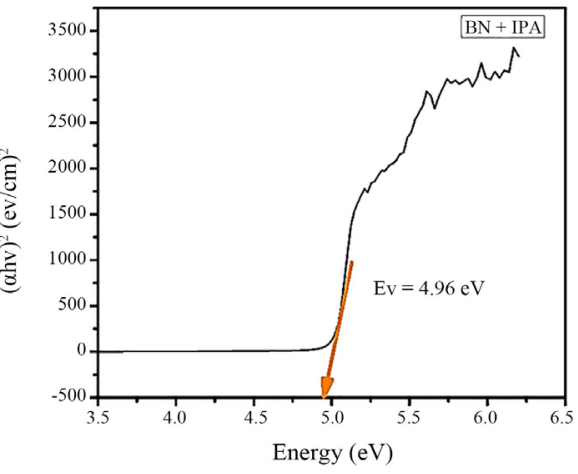

(b)

Figure 4. (a) Band Gap of multilayers of h-BN exfoliated in DMF; (b) Band Gap of multilayers of h-BN exfoliated in IPA.

\subsection{X-Ray Diffraction}

The Multilayers of h-BN were characterized under XRD to investigate the symmetry and phases. Comparative XRD patterns of h-BN multilayers exfoliated in IPA (red) and DMF (black) can be viewed in Figure 5. From the figure it reveals that diffraction peaks exhibit a slight variation of $\left(0.21^{\circ}\right)$. As the angle obtained by exfoliated BNNSs in DMF is $\left(27.39^{\circ}\right)$ while in IPA is $\left(27.17^{\circ}\right)$ [12]. In contrast to both samples no extra peaks were detected in the XRD pattern of exfoliated BNNSs. The intensity obtained by exfoliated BNNSs in DMF (452) is greater than exfoliated BNNSs in IPA (203) which can be analyzed from Figure 5.

The grain size of exfoliated BNNSs in DMF is $25.62 \mathrm{~nm}$ while in IPA is 43.73 $\mathrm{nm}$ which shows that $\mathrm{h}-\mathrm{BN}$ is exfoliated better in DMF with concerning to IPA. The peaks observed at angles $27^{\circ}$ in both samples confirms the formation of crystallographic planes (002), which is the indication of the formation of multilayers of BNNSs [13].

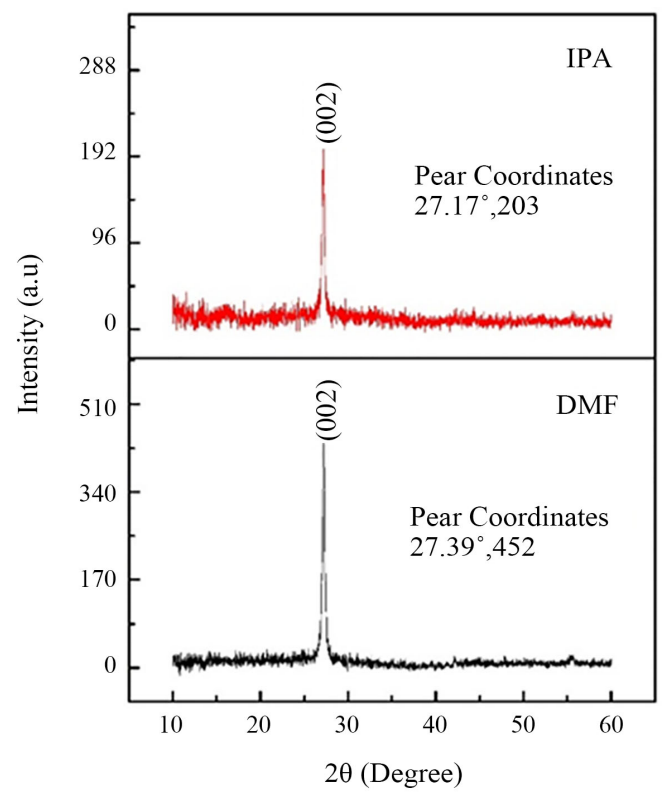

Figure 5. XRD pattern of h-BN exfoliated in IPA and DMF. 


\subsection{Scanning Electron Microscopy}

In SEM (Scanning electron microscopy) sample is scanned by using an energetic beam of electrons. Surface morphology, Chemical composition and material orientation such information is obtained by signals when beam of electron interacts with sample h-BN. In Figure 6(a), Figure 6(b) nanoflakes were pictured by SEM. These nanoflakes illustrating their plate like shape and size of the individual flake in the range of 200 nanometers which was observed in literature survey [14].

If the amount of incident electrons is higher than the amount of electrons evading from the sample, then the negative charge creates on sample at the point where the beam hits. This thing is called the charging effect [15] and it gives a range of scarce effects such as irregular contrast, image distortion and shift. That is why we see contrast in Figure 6(a), Figure 6(b) as this sample is drop cast on silicon substrate because of this we see black area where electron was distributed.

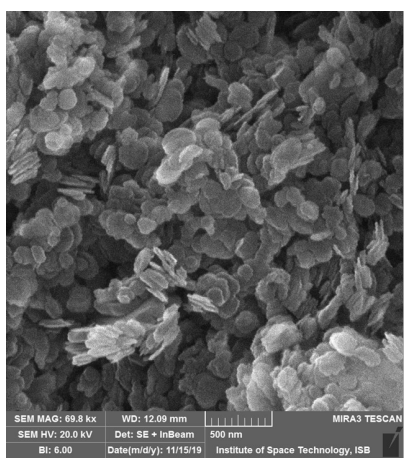

(a)

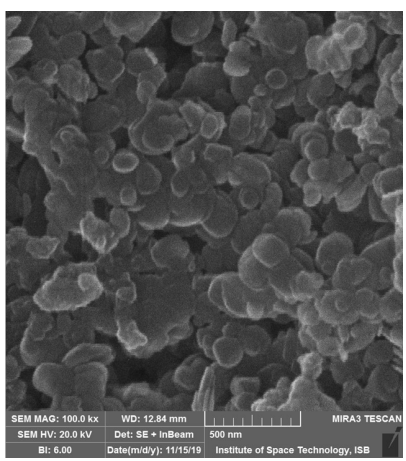

(b)

Figure 6. (a) Shows exfoliated h-BN in DMF at $500 \mathrm{~nm}$ and (b) Shows exfoliated h-BN in IPA at $500 \mathrm{~nm}$ respectively.

It was observed that Nano flakes obtained by sample exfoliated in DMF have fine distribution comparing to sample exfoliated in IPA.

\subsection{Dielectric Spectroscopy}

The exfoliated BN powder in DMF was drop cast on silicon substrate prepared as a function of thickness. The variation of thickness was 5, 10 and 15 drops. Similarly, exfoliated BN powder in IPA also drop cast on silicon substrate as a function of thickness. The variation of thickness was 5, 10 and 15 drops. By using an LCR meter capacitance was measured through which Dielectric constant is intended [16].

The formula to calculate the dielectric constant is

$$
k=\frac{C d}{A \varepsilon_{\circ}}
$$

In the above equation $\boldsymbol{C}$ shows capacitance, $\boldsymbol{d}$ depicts thickness, $\boldsymbol{A}$ is area of the sample and $\varepsilon_{0}$ is relative permittivity whose value is $8.85 \times 10^{-12}$ [17]. Exfoliated BN powder in DMF was drop cast on silicon substrate whose thickness 
variation can be observed with concerning for to no of drops same is observed in sample exfoliated in IPA [18].

Dielectric Constant is measured by using the formula and it was observed that its value increases with variation in thickness irrespective of solvent as in Table 1 and Table 2.

Table 1. Shows Dielectric constant value of h-BN exfoliated in DMF varying thickness.

\begin{tabular}{cccc}
\hline No of Drops & Thickness $(\mu \mathrm{m})$ & Capacitance $(\mathrm{pF})$ & Dielectric Constant \\
\hline 5 & 6 & 14 & 2.37 \\
10 & 8 & 16 & 3.61 \\
15 & 10 & 19 & 5.35 \\
\hline
\end{tabular}

Table 2. Shows Dielectric constant value of h-BN exfoliated in IPA varying thickness.

\begin{tabular}{cccc}
\hline No of Drops & Thickness $(\mu \mathrm{m})$ & Capacitance $(\mathrm{pF})$ & Dielectric Constant \\
\hline 5 & 7 & 10 & 2.25 \\
10 & 10 & 13 & 3.66 \\
15 & 13 & 16 & 5.85 \\
\hline
\end{tabular}

In Figure 7 histogram shown that as we increase the no of drops value of dielectric constant increases.

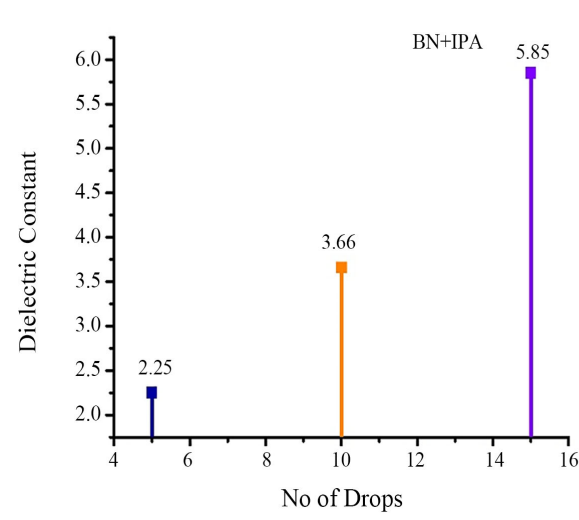

(a)

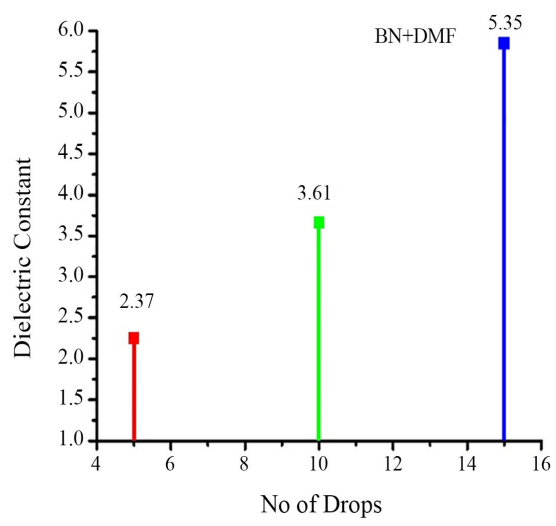

(b)

Figure 7. Shows variation in Dielectric constant (a) exfoliated sample in IPA and (b) exfoliated sample in DMF.

\section{Conclusion}

Increase in the value of dielectric constant was observed by increasing the thickness of hBNNs. Multilayers of h-BN were synthesized in DMF and IPA by using chemical exfoliation method. After successful exfoliation it was deposited on silicon substrate by drop cast method for characterization. Exfoliated samples in DMF and IPA have bandgap $5.39 \mathrm{eV}$ and $4.96 \mathrm{eV}$ respectively that is calculated by UV Visible spectroscopy. XRD patterns of h-BN exfoliated in DMF and IPA 
confirmed the mono-crystallite (002) nature of h-BN. SEM confirmed the formation of h-BN nanoflakes having size of $200 \mathrm{~nm}$. It was observed that nanoflakes obtained by sample exfoliated in DMF have fine distribution comparing to sample exfoliated in IPA. Capacitance is measured by using LCR meter. Using the capacitance value in a formula, the value of dielectric constant was calculated. The thickness of the samples were measured by using a profilometer. The variation was observed in value of dielectric constant as the thickness increases. h-BN nanocomposites with semi-conductor (metallic oxides) can be useful to obtain higher dielectric constant without varying thickness.

\section{Conflicts of Interest}

The authors declare no conflicts of interest regarding the publication of this paper.

\section{References}

[1] Koizumi, S., Murakami, T., Inuzuka, T. and Suzuki, K. (1990) Epitaxial Growth of Diamond Thin Films on Cubic Boron Nitride $\{111\}$ Surfaces by DC Plasma Chemical Vapor Deposition. Applied Physics Letters, 57, 563-565. https://doi.org/10.1063/1.103647

[2] Taniguchi, T., Kimoto, K., Tansho, M., Horiuchi, S. and Yamaoka, S. (2003) Phase Transformation of Amorphous Boron Nitride under High Pressure. Chemistry of Materials, 15, 2744-2751. https://doi.org/10.1021/cm021763j

[3] Wang, J., Ma, F. and Sun, M. (2017) Graphene, Hexagonal Boron Nitride, and Their Heterostructures: Properties and Applications. RSC Advances, 7, 16801-16822. https://doi.org/10.1039/C7RA00260B

[4] Bao, J., Jeppson, K., Edwards, M., Fu, Y., Ye, L., Lu, X. and Liu, J. (2016) Synthesis and Applications of Two-Dimensional Hexagonal Boron Nitride in Electronics Manufacturing. Electronic Materials Letters, 12, 1-16.

https://doi.org/10.1007/s13391-015-5308-2

[5] Hod, O. (2012) Graphite and Hexagonal Boron-Nitride Have the Same Interlayer Distance. Why? Journal of Chemical Theory and Computation, 8, 1360-1369. https://doi.org/10.1021/ct200880m

[6] Novoselov, K.S., Jiang, D., Schedin, F., Booth, T.J., Khotkevich, V.V., Morozov, S.V. and Geim, A.K. (2005) Two-Dimensional Atomic Crystals. Proceedings of the National Academy of Sciences of the United States of America, 102, 10451-10453. https://doi.org/10.1073/pnas.0502848102

[7] Park, J.H., Choi, S.H., Zhao, J., Song, S., Yang, W., Kim, S.M., Kim, K.K. and Lee, Y.H. (2016) Thickness-Controlled Multilayer Hexagonal Boron Nitride Film Prepared by Plasma-Enhanced Chemical Vapor Deposition. Current Applied Physics, 16, 1229-1235. https://doi.org/10.1016/j.cap.2016.03.025

[8] Gao, G., Gao, W., Cannuccia, E., Taha-Tijerina, J., Balicas, L., Mathkar, A., Narayanan, T.N., Liu, Z., Gupta, B.K., Peng, J., Yin, Y., Rubio, A. and Ajayan, P.M. (2012) Artificially Stacked Atomic Layers: Toward New Van der waals Solids. Nano Letters, 12, 3518-3525. https://doi.org/10.1021/nl301061b

[9] Zhang, B., Wu, Q., Yu, H., Bulin, C., Sun, H., Li, R., Ge, X. and Xing, R. (2017) High-Efficient Liquid Exfoliation of Boron Nitride Nanosheets Using Aqueous Solution of Alkanolamine. Nanoscale Research Letters, 12, 596.

https://doi.org/10.1186/s11671-017-2366-4 
[10] Zhou, K.G., Mao, N.N., Wang, H.X., Peng, Y. and Zhang, H.L. (2011) A Mixed-Solvent Strategy for Efficient Exfoliation of Inorganic Graphene Analogues. Angewandte Chemie International Edition, 50, 10839-10842. https://doi.org/10.1002/anie.201105364

[11] Lin, Y., Williams, T.V. and Connell, J.W. (2010) Soluble, Exfoliated Hexagonal Boron Nitride Nanosheets. The Journal of Physical Chemistry Letters, 1, 277-283. https://doi.org/10.1021/jz9002108

[12] Cui, Z., Oyer, A.J., Glover, A.J., Schniepp, H.C. and Adamson, D.H. (2014) Large Scale Thermal Exfoliation and Functionalization of Boron Nitride. Small, 10, 2352-2355. https://doi.org/10.1002/smll.201303236

[13] Rafiei-Sarmazdeh, Z., Jafari, S.H., Ahmadi, S.J. and Zahedi-Dizaji, S.M. (2016) Large-Scale Exfoliation of Hexagonal Boron Nitride with Combined Fast Quenching and Liquid Exfoliation Strategies. Journal of Materials Science, 51, 3162-3169. https://doi.org/10.1007/s10853-015-9626-4

[14] Xu, M., Liang, T., Shi, M. and Chen, H. (2013) Graphene-Like Two-Dimensional Materials. Chemical Reviews, 113, 3766-3798. https://doi.org/10.1021/cr300263a

[15] Shaffner, T.J. and Van Veld, R.D. (1971) “Charging” Effects in the Scanning Electron Microscope. Journal of Physics E, 4, 633-637. https://doi.org/10.1088/0022-3735/4/9/002

[16] Kim, S.M., Hsu, A., Park, M.H., Chae, S.H., Yun, S.J., Lee, J.S., Cho, D.H., Fang, W., Lee, C., Palacios, T., Dresselhaus, M., Kim, K.K., Lee, Y.H. and Kong, J. (2015) Synthesis of Large-Area Multilayer Hexagonal Boron Nitride for High Material Performance. Nature Communications, 6, 8662. https://doi.org/10.1038/ncomms9662

[17] Laturia, A., Van de Put, M.L. and Vandenberghe, W.G. (2018) Dielectric Properties of Hexagonal Boron Nitride and Transition Metal Dichalcogenides: From Monolayer to Bulk. NPJ2D Materials and Applications, 2, 1-7.

https://doi.org/10.1038/s41699-018-0050-x

[18] Ahmed, F., Heo, S., Yang, Z., Ali, F., Ra, C.H., Lee, H.I., Taniguchi, T., Hone, J., Lee, B.H. and Yoo, W.J. (2018) Dielectric Dispersion and High Field Response of Multilayer Hexagonal Boron Nitride. Advanced Functional Materials, 28, Article ID: 1804235. https://doi.org/10.1002/adfm.201804235 